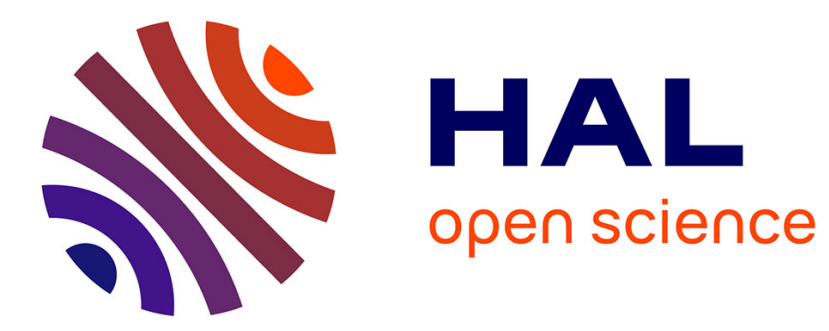

\title{
Warm Dense Matter through Classical Molecular Dynamics.
}

Annette Calisti, Sandrine Ferri, Mathieu Marciante, Bernard Talin

\section{To cite this version:}

Annette Calisti, Sandrine Ferri, Mathieu Marciante, Bernard Talin. Warm Dense Matter through Classical Molecular Dynamics.. High Energy Density Physics, 2014, 13, pp.1-8. 10.1016/j.hedp.2014.07.002 . hal-01053438

\section{HAL Id: hal-01053438 https://hal.science/hal-01053438}

Submitted on 31 Jul 2014

HAL is a multi-disciplinary open access archive for the deposit and dissemination of scientific research documents, whether they are published or not. The documents may come from teaching and research institutions in France or abroad, or from public or private research centers.
L'archive ouverte pluridisciplinaire HAL, est destinée au dépôt et à la diffusion de documents scientifiques de niveau recherche, publiés ou non, émanant des établissements d'enseignement et de recherche français ou étrangers, des laboratoires publics ou privés. 


\title{
Warm Dense Matter through Classical Molecular Dynamics
}

\author{
A. Calisti ${ }^{\mathrm{a}, *}$, S. Ferri ${ }^{\mathrm{a}}$, M. Marciante ${ }^{\mathrm{b}}$, B. Talin ${ }^{\mathrm{a}}$ \\ ${ }^{a}$ Aix-Marseille Université, CNRS, PIIM, UMR7345, Campus Saint Jérôme, 13397 Marseille Cedex 20, France. \\ ${ }^{b}$ LASIM, UMR 5579 CNRS / UCBL, Domaine Scientifique de la Doua, Université Claude Bernard Lyon 1, 69622 Villeurbanne cedex, France
}

\begin{abstract}
A classical Molecular Dynamics code has been developed to simulate dense plasmas i.e. neutral systems of interacting ions and electrons. Our goal is to design a tool that relies on a reduced set of microscopic mechanisms in order to obtain solutions of complex time dependent n-body problems and to allow an efficient description of the plasma states between classical high temperature systems to strongly coupled plasmas. Our present objective is an attempt to explore the behavior of such a classical approach for typical conditions of warm dense matter. We calculate the dynamic structure factor in warm dense beryllium by means of our molecular dynamics simulations. The results are then compared with those obtained within the framework of the random phase approximation (RPA).
\end{abstract}

Keywords:

Warm dense matter, Classical molecular dynamics, Dynamic structure factors.

\section{Introduction}

The purpose of this work is to investigate the dynamical properties of electrons in warm dense plasmas using classical molecular dynamics techniques. In dense plasmas, electron dynamics is revealed in X-ray Thomson scattering experiments developed to diagnose electron temperature and density, see Ref. [1] and references therein. Schematically, a monochromatic incident X-rays, which can penetrate dense and/or compressed matter, couples to electron density fluctuations and the scattered spectrum shows a central peak surrounded by wings mainly related to the free electron dynamics characteristics. If the length scale of the electron density fluctuations measured in the scattering experiment is larger or shorter than the screening length of the interaction potential of the electrons, the collective or individual behavior of the electrons will be accessed, respectively. Using Thomson scattering as a diagnostic requires a proper theoretical treatment of the dynamic structure factor (DSF) for the interpretation of spectra. There is still ongoing research for cases where the warm dense matter (WDM) occurs, which is a complicated state of matter: electron degeneracy and strong ion-ion coupling mean that neither classical plasma nor solid matter formulations that use the usual approximations satisfactorily apply. In this context, simulation is a reasonable way to predict the scattering spectra.

As other approaches to complexity, e.g., quantum molecular dynamics (QMD), classical molecular dynamics (MD) for two component plasmas (TCP) has to be developed within a framework of constraints and approximations either grounded in theoretical formulations or for the sake of computational feasibility. TCP-MD proposed in this work [2,3], does not make use of the Born-Oppenheimer approximation and relies on a minimum set of microscopic mechanisms implemented into a numerical code designed to obtain numerical solutions of complex time-dependent $\mathrm{N}$-body problems.

The advantage of MD is to allow an efficient description of the transition between classical high temperature systems and strongly coupled plasmas. Conditions at which quantum effects would prevail are not neatly defined. Bellow we choose to explore the transition between classical and partially degenerate plasmas by comparing TCPMD results with those provided by other numerical and theoretical methods. For this purpose it is necessary to use the

\footnotetext{
${ }^{*}$ Corresponding author

Email address: annette.calisti@univ-amu.fr (A. Calisti )
} 
same simulation model over the whole density range. For TCP-MD, all the particles considered are point particles, thus the main constraint is the need to choose potentials that avoid the short range collapse of ion-electron pairs. The first advantage of this choice is taken to introduce known ad hoc quantum characteristics of isolated ions, e.g., the ionization energy or the diffusive properties of an ion for colliding electrons. The second advantage is that one gains the ability to describe the ion-electron coupling accounting for mixtures of ions undergoing changes of their ionization stages. Note that the coupling of electrons with radiation is ignored. Within this classical scheme the energy of electrons is continuous. The lowest energy of an electron depends on all the charges including the closest ion. The notion of discrete energy for the ionic excited states is here replaced by its continuous equivalent. Depending on its total energy and its nearest neighbor ion, an electron is either trapped or free.

One postulates that molecular dynamics of a neutral system of ions and electrons with soft potentials, has stable solutions. Ignoring possible numerical drifts, stable here means that inside the simulation cell, temperature and potential energy fluctuate around averages. The way to drive the system into such a state, i.e., to reach a suitable phase space trajectory, is in itself a major problem. The simplest method is to initiate the system into a unequilibrated state then use an empirical procedure that constrains its evolution to approach an equilibrium state. The possibility of using such a procedure takes advantage of TCP-MD, which is designed to follow the evolution of a system of ions and electrons out of equilibrium.

Below the following points will be discussed: First, the simulation model based on approximate charge-charge potentials together with a three-body collisional ionization/recombination mechanism is presented. Second, the technique used to obtain the collective behavior of the free electrons from the TCP-MD is described. Finally, the model and techniques are illustrated for the case of warm dense beryllium.

\section{Two component plasma molecular dynamics simulations.}

Classical molecular dynamics for two component plasmas, i.e., for mixture of interacting ions and electrons has proven useful, see for example [4, 5, 6]. It complements QMD to span the whole domain of dense plasmas, from solid to high energy matter. The specificity of the present model relies on the choice of the charge-charge potentials and a mechanism of ionization/recombination allowing one to model systems with ions in variable ionization states. The MD simulations reported below are standard in the sense that a finite number of particles is considered in a cubic cell. The velocity-Verlet algorithm is used to propagate the dynamics. The time step has to be chosen to be sufficiently small enough to allow a proper description of electron motion. This requirement leads to a technical difficulty since the total simulated time necessary to obtain relevant statistics is governed by the mobility of ions of the system. Here we apply periodic boundary conditions to the cubic simulation cell together with the minimal image convention convention to simulate an infinite system. In the following, several useful statistical data are sampled when the system is considered in a stationary state.

\subsection{Soft potentials}

The ion-ion and electron-electron interactions are taken to be

$$
V_{12}(r)=Z_{1} Z_{2} e^{2} e^{-r / \lambda} / r
$$

where $Z_{1} Z_{2}$ is positive. For practical purposes, the Coulomb interactions have been screened at a distance $\lambda \simeq L / 2$, of the order of the cubic cell size $L$. This screening is compatible with the usual periodic boundary conditions in the MD simulation and given that the interactions between charges introduce a physical screening at much shorter distances, it does not affect any of the properties considered in this work. This point has been checked numerically by increasing the box size. The electron-electron potential does not account for degeneracy. The choice of a repulsive potential that excludes short distance location of particles of the same sign is required to guarantee the exchange of the mechanical energy between positive and negative charges. The electron dynamics investigated is not that of a jellium model [7,8] and, thus, has to account for the ion potential wells.

In contrast, electron-ion interactions are attractive and therefore configurations involving electron-ion distances of the order of the de Broglie wavelength or shorter have to be considered. At such distances, the Coulomb interaction must be regularized. A standard regularized potential is as follow, [9]

$$
V_{i e}(r)=-Z_{i} e^{2}\left(1-e^{-r / \delta}\right) e^{-r / \lambda} / r
$$


where $\delta$ is the short range regularization parameter. Regularization provides well-defined classical physics for opposite sign charge systems, and allows application of the N-body methods of classical statistical mechanics.

A great deal of work on plasmas has been performed with the help of pseudo potentials, [10, 9, 11]. Owing to the temperature and density domain considered in the present work, the choice of a potential is guided by the study of the evolution of ion charge populations. For this purpose, $\delta$ depends on the instantaneous ionization state in order to fit the ionization energies of the various ion stages. This choice would not be suitable for electron temperatures much higher than the highest ionization energy, leading to a plasma of fully ionized ions and electrons. In a potential given by Eq. 2 the minimum energy of an electron located at the same position as an ion of charge $Z$ is $Z e^{2} / \delta$. In what follows, the kinetic energy required to overcome this minimum potential energy, i.e. $Z e^{2} / \delta$, will be considered as the ionization energy for an electron trapped by an ion of charge $Z$. In this model, the zero temperature limit is defined as a state where each ion has a unit charge and possesses a single electron located at the bottom of its potential well without relative velocity with respect to the ion.

Other parameters of interest are the average charge-charge distance, $r_{0}=(3 / 4 \pi n)^{1 / 3}$, defined in terms of the charge density $n$, the electron thermal velocity $v_{0}=\left(k_{B} T_{e} / m_{e}\right)^{1 / 2}$, the ion coupling constant $\Gamma=\frac{<Z>^{2} e^{2}}{r_{0} k_{B} T}$ where $<>$ is an ensemble average, the thermal de Broglie wavelength $\Lambda_{e}=h /\left(2 \pi m_{e} k_{B} T_{e}\right)^{1 / 2}$, the Debye length $\lambda_{D}=\left(k_{B} T_{e} / 4 \pi n_{e} e^{2}\right)^{1 / 2}$ and the electron degeneracy parameter $\Theta_{e}=\frac{2 m_{e} k_{B} T_{e}}{\hbar^{2}}\left(3 \pi^{2} n_{e}\right)^{-2 / 3}$. Molecular dynamics simulations of two component plasmas (TCP-MD) are carried out using $N$ electrons and $N / Z_{i}$ ions. The chosen simulation protocol implies that the electronic density is a fluctuating quantity.

\subsection{Collisional ionization recombination protocol in classical MD}

Usual collisional models [12] for ionization/recombination in plasmas assume that electrons follow well-defined trajectories given by their impact parameter and their velocity. Averages are performed to calculate the ionization and recombination rates and the resulting equilibrium ion charge distributions. This approximation cannot be considered as relevant for dense plasmas for which the notion of binary electron-ion collision becomes inappropriate. On average, for a neutral plasma there are $Z_{i}$ electrons per ion and both the trajectory and the energy of an electron are nonlinear functions of all surrounding charges. In an attempt to describe multi-collisional processes in dense plasmas, we recently developed more suitable concepts practical to MD simulations. The notion of a shell has been introduced to characterize the near plasma environment of ions. The shell belonging to a particular ion is composed of its two nearest neighbor electrons, denoted first nearest $(F N e)$ and second nearest $(S N e)$ electron. During a time step $\left[t_{i}, t_{i}+\delta t\right]$, depending on the local configuration space of the plasma (mutual nearest neighbors criterion between the ion and its $\mathrm{FNe}$ ) and on the total energy of the two neighbour electrons, the shell is labeled hot, cold or inactive. The electron total energy criterion takes into account the whole complexity of the potential energy surface around the ion including the ionization energy lowering at a local level due to the surrounding charges.

A hot shell around one ion starts a ionization process of this ion while for a cold shell a recombination of the $F N e$ electron occurs. This means that during the time step $\left[t_{i}, t_{i}+\delta t\right]$, an electron appears or disappears and the ion charge increases or decreases by one unit. After this instantaneous event all the involved particles continue evolving according to dynamics of the simulation. In this way an electron appearing at the same place as the ion at the time of an ionization process, is progressively accelerated by its surrounding environment, increasing during this time its potential energy. Another discontinuous event applied to the same ion is prohibited for the time it takes for an electron to cross a distance equal to the de Broglie wavelength. This delay is intended to account for time uncertainty of the ionization process beginning. The recombination process follows the same scheme, i.e., an instantaneous event where an electron disappears and the ion charge decreases by one. The kinetic energy loss of the recombining electron is transferred to the $\mathrm{SNe}$. Then, the process is completed by an energy redistribution among the remaining charges within the normal simulation process. The actual ionization or recombination of an ion lasts until a new ionization or a recombination becomes allowed.

Two remarks have to be made: First, the particular forms of the chosen interaction potentials do not allow the conservation of the total energy during the collisional ionization/recombination process. This leads to non-negligible energy drift during the equilibration step of the simulation, when all thermodynamic properties, as well as the mean ion charge, are far from equilibrium. During this initial step the system is driven toward equilibrium using a thermostat and is not supposed to be used for any measurements. Once the system has reached an equilibrium state, the ionization 
and recombination rates become equals and the effect of the process reduces to small residual energy fluctuations. At the same time, the happening of the process become far less frequent than it was in the equilibration step. Second, this imperfect mechanism allows an efficient evolution of the initial non equilibrated ion - electron system towards equilibrium.

\subsection{Statistical data}

In order to extract statistical information from the simulated system a few distributions and time-dependent functions are calculated. These data allow one to check the system behavior as well.

The pair correlation functions carry information about the system structure, i.e., the way charges are statistically located with respect to the other ions. These functions are often interpreted in terms of screening mechanisms as an ionic system built with screened forces between ions can have a similar structure than the one obtained from TCP simulation. Hereafter, the ionic pair correlation functions are compared to those provided by finite-temperature density functional theory molecular dynamics (FT-DFT-MD) simulations [13] and by screened Coulomb one component plasma (Yukawa OCP) simulations. FT-DFT-MD simulations, or ab initio simulations, aim to describe fully interacting quantum systems. They include ionic correlations as well as the quantum behavior for the electrons. In the Yukawa model, only the ions are explicitly considered, and the electrons are treated as a polarizable background. The Yukawa potential is then given by:

$$
V_{i i}(r)=\frac{Z_{i}^{2} e^{2}}{r} e^{-\kappa r}
$$

To describe the partially degenerate electrons in WDM, the inverse screening length $\kappa$ should be calculated by $\kappa^{2}=\left(4 e^{2} m_{e} / \pi \hbar^{3}\right) \int d p f_{e}(p)$ with $f_{e}(p)$ the Fermi distribution. With this definition, the Thomas-Fermi screening length is given by $\lambda_{T F}=\sqrt{\frac{k_{B} T_{F}}{6 \pi n_{e} e^{2}}}$ with $T_{F}=\frac{\hbar^{2}\left(3 \pi^{2} n_{e}\right)^{2 / 3}}{2 k_{B} m_{e}}$. In the following, the two limiting cases corresponding to the classical Debye-Hückel law and Thomas-Fermi screening are presented.
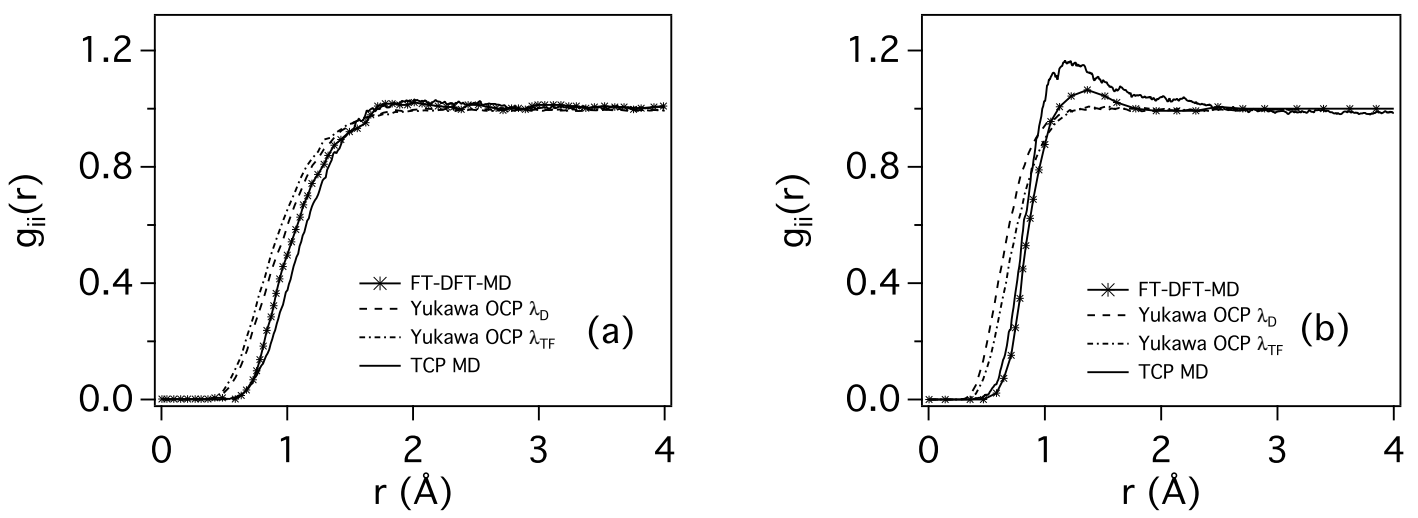

Figure 1: Ion-ion pair distribution functions in warm dense beryllium at (a): $\rho_{0}=1.85 \mathrm{~g} / \mathrm{cm}^{-3}$ and $T_{e}=T_{i}=12 \mathrm{eV}$ and (b): $\rho_{0}=5.5 \mathrm{~g} / \mathrm{cm}^{-3}$ and $T_{e}=T_{i}=13 \mathrm{eV}$. The ion charge states (for the Yukawa OCP simulation) are $Z=2.17$ and $Z=2.24$ for (a) and (b), respectively.

It can be seen in Fig.1 that our model reproduces rather well the interparticle spacing and the maximum in the distribution. The Yukawa model, which treats the electrons within linear response, underestimates ion-ion repulsion at small distances. It can be also noticed that our model reproduces qualitatively the results obtained with "FT-DFTMD”. The remaining differences, which appear to be of the same order as the differences between the two OCP models, could be attributed to the fact that we neglect any electron degeneracy effects. 
The velocity distribution functions of each kind of particles in a pure classical TCP without ionization/recombination protocol is Maxwellian. In this work, the electron velocity distribution function is non-Maxwellian as ionization/recombination generates excited electrons with non-thermal velocities. When the electronic temperature decreases, it would be necessary to take into account the Pauli exclusion principle that gives rise to a Fermi-Dirac distribution instead of a Maxwell-Boltzmann one. This is not done here and the consequences will be commented on in Sec. 3. The electron energy distribution function is plotted in Fig.2. The distribution function of negative energies is associated with trapped or free slow electrons. Associated to the knowledge of total number of electrons in the simulation box, this function allows us to estimate the density of free electrons in our simulations. Moreover, the formation of the negative wing helps to follow the evolution of the TCP system towards stability.

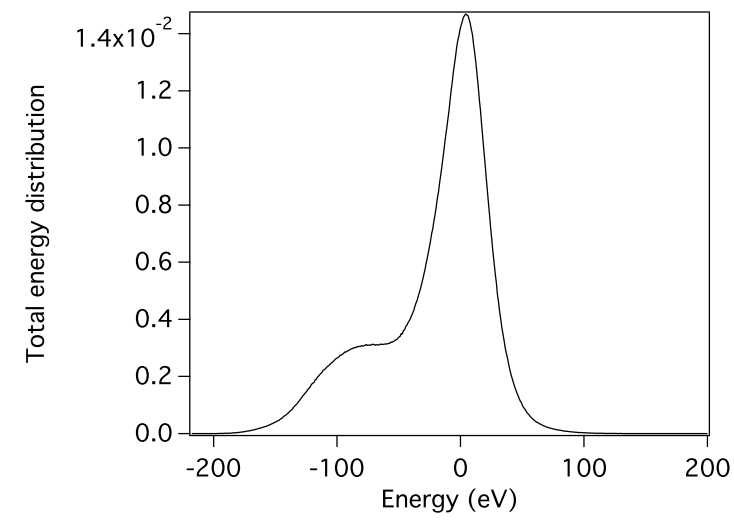

Figure 2: Electron energy distribution for a beryllium plasma at $\rho_{0}=1.85 \mathrm{~g} / \mathrm{cm}^{-3}$ and $T_{e}=T_{i}=12 \mathrm{eV}$.

Considering a binary system reduced to a single electron bounded in the potential well of an ion $i$ of charge $Z_{i}$, the minimum electron potential energy is the ionization energy parameter $E_{i}$ of the model. If a second ion $j$ of charge $Z_{j}$ is added to this system, provided the inter-ion distance is small enough, the energy barrier seen by the electron to escape the potential of ion $i$ will be significantly lowered in the direction of ion $j$. Similar reasoning holds if one adds an electron instead of an ion to the binary system, and this leads to a lowering of the potential well seen by the bound electron in the opposite direction of the additional electron. In this way, the model naturally describes the ionization energy as a function of the local configuration of charges, which statistically results in an ionization potential lowering.

The dynamic behavior of the free electrons can be investigated through the density-density dynamic structure factor (DSF). It is given by:

$$
\begin{gathered}
S(\vec{k}, \omega)=\frac{1}{2 \pi N} \int_{-\infty}^{+\infty} e^{i \omega t}<\rho(\vec{k}, t) \rho(-\vec{k}, 0)>d t \\
\rho(\vec{k}, t)=\sum_{i=1}^{N} e^{i \vec{k} \cdot \vec{r}_{i}(t)}
\end{gathered}
$$

The DSF is related to the dielectric function $\epsilon(\vec{k}, \omega)$ via the fluctuation-dissipation theorem (FDT):

$$
S(\vec{k}, \omega)=\frac{\hbar k^{2} \operatorname{Im} \epsilon^{-1}(\vec{k}, \omega)}{n 4 \pi^{2} e^{2}\left[1-e^{-\beta \hbar \omega}\right]}
$$

This function does not possess any symmetry and satisfies the detailed balance relation, $S(-\vec{k},-\omega)=e^{-\beta \hbar \omega} S(\vec{k}, \omega)$. In classical MD simulations, identifying the Heisenberg operator $\vec{r}_{i}(t)$ with the position of the $\mathrm{i}^{\text {th }}$ particle, we get:

$$
R(\vec{k}, \omega)=\frac{k^{2}}{n 4 \pi^{2} e^{2} \beta \omega} \operatorname{Im} \epsilon^{-1}(\vec{k}, \omega)
$$




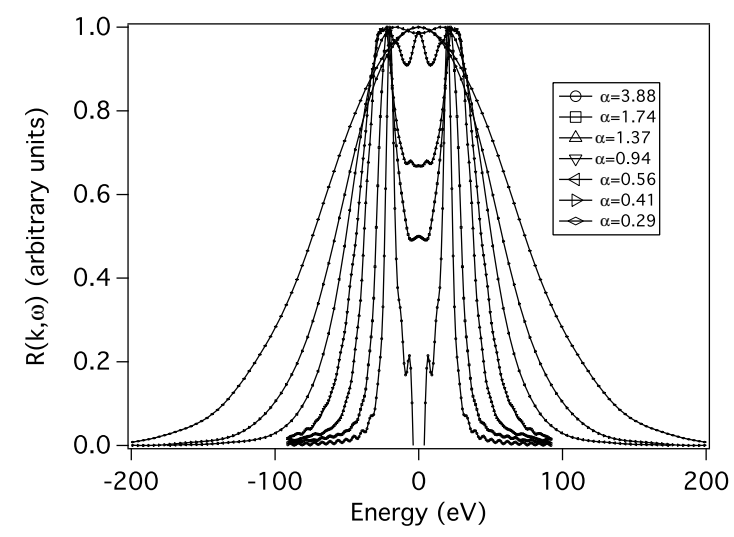

Figure 3: $R$ Function for different values of the scattering parameter and for the same conditions as Fig.2.

This function reveals the electron density fluctuations on a length scale given by $\lambda_{\text {scat }}=2 \pi / k$ with $k=|\vec{k}|$.

The dimensionless scattering parameter, $\alpha=1 / k \lambda_{s}$ which compares $\lambda_{\text {scat }}$ with the screening length $\lambda_{s}$ is defined to characterize the scattering regime. At $\alpha>1$, in the collective scattering regime, the density fluctuations at a scale larger than the screening length are probed, while at $\alpha<1$, in the non-collective scattering regime, the density fluctuations of individual electrons are resolved. Depending on the scattering regime, the scattered spectra show Compton or plasmon features that are related to individual or collective charge properties, respectively.

As an example, we have plotted in Fig. 3, the $R$ function corresponding to the density fluctuation of the free electrons in a Be plasma at $\rho_{0}=1.85 \mathrm{~g} / \mathrm{cm}^{-3}$ and $T_{e}=T_{i}=12 \mathrm{eV}$. It can be seen that the behavior of the $R$ function versus $\alpha$ reproduces correctly the description given in ref. [14]. For $\alpha>1$ the function presents two symmetric well pronounced maxima and as $\alpha$ decreases, the dip between the two maxima is progressively filled to reach a Gaussian shape at $\alpha=0$.

The DSF, $S(\vec{k}, \omega)$, (see Fig. 4 ) is related to the $R$ function by:

$$
S(\vec{k}, \omega)=\frac{\hbar \beta \omega}{1-e^{-\hbar \beta \omega}} R(\vec{k}, \omega)
$$

which approximates Eq. 6 assuming that the main quantum effects are due to the detailed balance.

The DSF as the basic input for the Thomson scattering cross-section is directly related to the spectrally resolved $\mathrm{x}$-ray Thomson scattering measurements [1] which are widely used as diagnostics to infer dense plasma parameters such as electronic density and temperature. A more detailed analysis of our results is provided in Sec. 3 for conditions corresponding to two recent experiments performed on beryllium $[15,16]$.

\section{Application to beryllium}

For a few years, the behavior of warm dense beryllium has been an issue of interest from both a theoretical and experimental point of view $[15,16,17,18,19,20,13]$. The recent possibility to carry out comparisons with different approaches motivates our interest to develop a pure classical investigation based on classical MD. TCP MD provides straightforward access to electron dynamics and the possibility to observe free electron plasmons for the interpretation of Thomson scattering experiments. Two WDM conditions have been investigated so far, labeled in literature as the un-compressed and compressed beryllium with the density and temperature conditions; $\rho_{0}=1.85 \mathrm{~g} / \mathrm{cm}^{-3}$ and $T_{e}=T_{i}=12 \mathrm{eV}$ and $\rho_{0}=5.5 \mathrm{~g} / \mathrm{cm}^{-3}$ and $T_{e}=T_{i}=13 \mathrm{eV}$, respectively. The comparisons rely on the dynamic structure factor of free electrons $S_{e e}(\vec{k}, \omega)$.

Warm dense matter conditions result in a de Broglie wavelength of the order of the average distance between particles. This often motivates the implementation of a regularized electron-electron potential intended to represent electronelectron exchange in numerical simulations. With our ionization/recombination protocol, this model leads to unphysical results. With a large regularization length all the electrons become uncoupled. The strong ion-electron coupling 


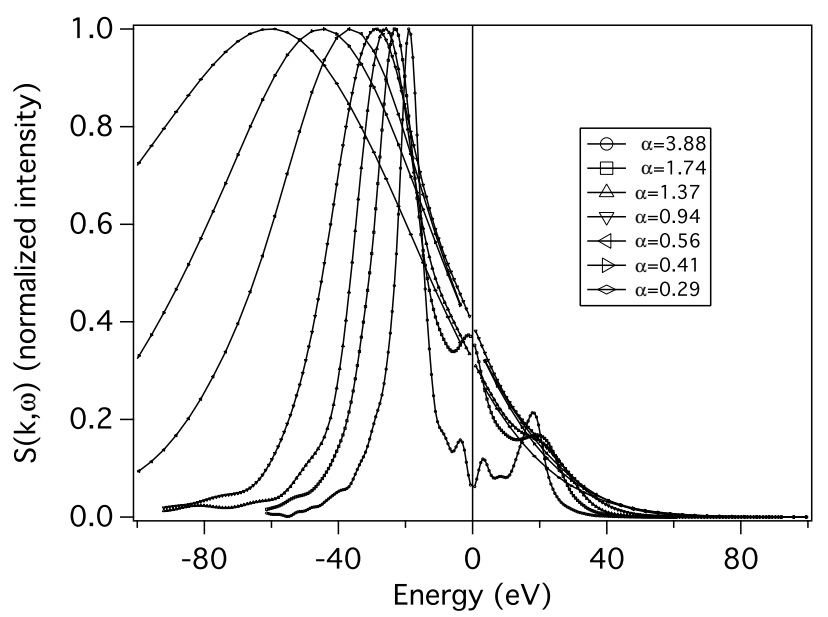

Figure 4: Structure factor, $S$, for different values of the scattering parameter and for the same conditions as Fig.2.

is no longer in competition with the electron-electron coupling for those electrons falling into the ion potential wells. Charge coupling insures the mechanism of energy exchange between particles and leads to plasma simulations that reach equilibration to a stable state.

\subsection{Un-compressed beryllium}

Here, the TCP MD results are presented for plasma conditions corresponding to those of the first observation of plasmons in solid-density plasmas [15]. The mass density is $\rho_{0}=1.85 \mathrm{~g} / \mathrm{cm}^{-3}$ and the temperature is set to 12 $\mathrm{eV}$ in accordance with the theoretical best-fit of the experimental data provided by authors. The experiment was designed to observe collective x-ray forward scattering spectra at an average scattering angle of $\theta=40^{\circ}$ with the probe wavelength $\lambda_{0}=0.42 \mathrm{~nm}$. In the nonrelativistic limit and for small momentum transfers, the wave vector $\vec{k}$ is related to the scattering angle through:

$$
k=|\vec{k}|=\frac{4 \pi}{\lambda_{0}} \sin (\theta / 2)
$$

where $\lambda_{0}$ is the wavelength of the incident wave. For MD simulations performed in a cubic box of side $L$, due to periodic boundary conditions, $\vec{k}$ must satisfy:

$$
k_{x, y, z}=n_{x, y, z} \frac{2 \pi}{L}
$$

where $n_{x, y, z}$ is an integer number. In this work, the DSF has been calculated for different values of $k$ in order to span the values of $\alpha=\frac{1}{k \lambda_{D}}$ from 0.2 to 3.88, from non collective to collective scattering regimes. The smallest value of $k$ reachable in our simulation is determined by the highest number of simulated particles $N$ (constraining $L$ through the plasma mean density $N / L^{3}$ ) allowing one to keep the simulations performed to a reasonable time.

According to these constraints, TCP-MD simulations have been performed with 220 atoms of beryllium. We started the simulations with an electronic density corresponding to a mean charge $Z_{b}=2$. After an equilibration step controlled by imposing a temperature of $12 \mathrm{eV}$, we ensure that an equilibrium state has been reached by checking the stationarity of the total energy and charge distributions. The equilibrium state is reached for $n_{e}=2.68 \times 10^{23} \mathrm{~cm}^{-3}$ and $Z_{b}=2.17$. This corresponds to charge coupling parameters $\Gamma_{e}=1.25$ and $\Gamma_{i}=4.53$ for electrons and ions respectively, and a degeneracy parameter $\Theta_{e}=0.79$. These values, $\Gamma_{i, e}>1$ and $\Theta_{e}<1$ indicate that the plasma statistical data should show strong correlation and degeneracy effects. The free electron DSF obtained by TCP-MD is plotted in Fig.5(a) and (b) for $\alpha=3.88$ and $\alpha=0.65$ respectively. They are compared with those obtained in the same way by OCP MD simulations in which only interacting electrons are considered and with the random phase approximation (RPA) calculations in which the dielectric function is calculated for a one component plasma of free 

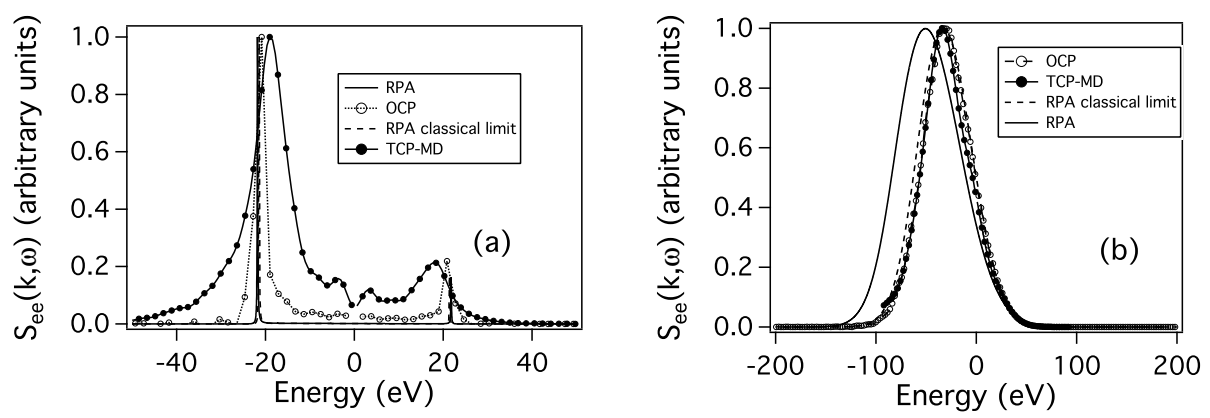

Figure 5: Dynamic structure factor for (a): $\alpha=3.88$ and (b): $\alpha=0.65$ plotted for $n_{e}=2.68 \times 10^{23} \mathrm{~cm}^{-3}$ and $T_{e}=12 \mathrm{eV}$.

electrons without interactions [21]. As the TCP-MD simulations are classical simulations, the RPA results in the classical limit are also plotted for comparisons. The DSF plotted in Fig.5(a) corresponds to free electron density fluctuations in the collective scattering regime and is mainly affected by correlations and collective effects. It can be seen that the plasmon peak obtained by TCP-MD is much wider and shifted to slightly lower frequencies than those obtained by RPA. At this value of $\alpha$, degeneracy effects are negligible (the differences between RPA and RPA in the classical limit results are small). The main differences between the RPA calculations and MD simulations concern interactions between charges which results in a broadening and shift toward the low frequencies of the plasmon peak. These effects concern the electron-electron interactions (compare RPA results with interacting electron OCP simulation) and are strongly enhanced accounting for ion-electron interactions (TCP-MD results). Similar results have already been obtained previously in Ref. [13].

In contrast, for $\alpha=0.65$, the DSF displayed in Fig.5(b) is measured in the non-collective scattering regime and accesses the properties of individual electrons as the shape of the DSF reflects the velocities of the electrons in the direction of the scattering vector $\vec{k}$. This will permit one to evaluate the importance of the electron degeneracy effects. It can be seen that our result differs from the RPA calculation due to the presence of degenerate electrons. The RPA calculation in the classical limit agrees very well with our results. The effects of interactions between charges begin to be negligible.

\subsection{Compressed beryllium}

The results presented in this section correspond to observations of both the inelastic Compton and plasmon scattering spectra from shock-compressed dense matter [16]. The mass density is $\rho_{0}=5.5 \mathrm{~g} / \mathrm{cm}^{-3}$ and the temperature is $13 \mathrm{eV}$. In the experiment, two scattering angles were chosen to probe the density fluctuations in both scattering regimes, i.e., the collective regime with $\theta=25^{\circ}$ and non-collective regime with $\theta=90^{\circ}$. The probe wavelength here is $0.2 \mathrm{~nm}$.

In order to fulfill Eq. 9 and Eq. 10 with small enough values of $k$, the TCP-MD simulations have been performed with 200 atoms of beryllium. We started the simulations with a temperature of $13 \mathrm{eV}$ and an electronic density corresponding to a mean charge $Z_{b}=3$. After the equilibration phase, we reached an equilibrium state with the parameters: $n_{e}=8.21 \times 10^{23} \mathrm{~cm}^{-3}$ and $Z_{b}=2.24$. This corresponds to charge coupling parameters $\Gamma_{e}=1.67$ and $\Gamma_{i}=6.41$ for electrons and ions, respectively, and a degeneracy parameter $\Theta_{e}=0.41$.

Here again, it can be seen in Fig.6(a) that the plasmon peak location and width are modified by the density effects. Figure 6(b) clearly demonstrates that the electron are mainly degenerate and thus our model is not appropriate to simulate this case with $\Theta_{e}=0.41$ and $\alpha<1$.

\section{Discussion}

For a better understanding of the different results, an analysis of the maximum position $\Delta E$ of $S_{e e}(k, \omega)$ as a function of $k$ has been performed. 

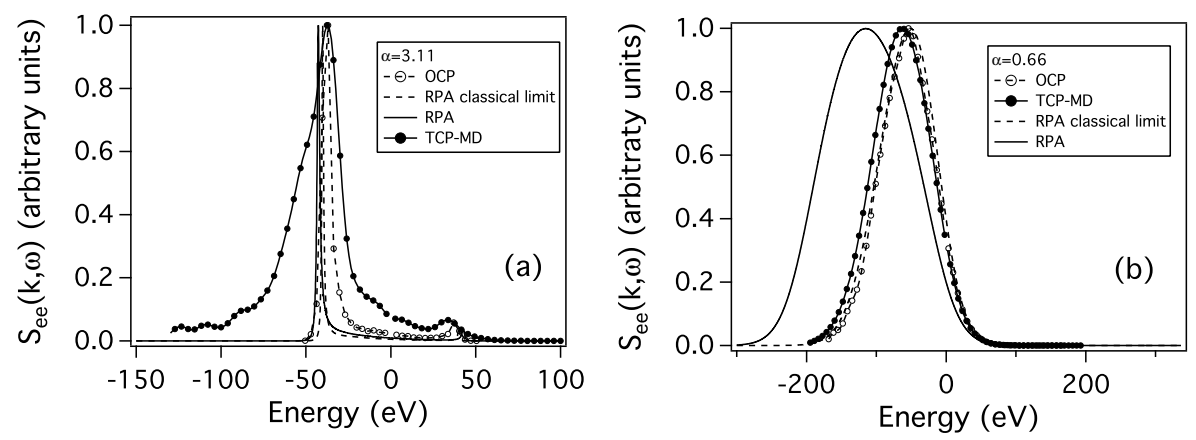

Figure 6: Dynamic structure factor for (a): $\alpha=3.11$ and (b): $\alpha=0.66$ plotted for $n_{e}=8.21 \times 10^{23} \mathrm{~cm}^{-3}$ and $T_{e}=13 \mathrm{eV}$.

We recall that for $\alpha<1$, the density fluctuations of individual electrons are resolved and the Compton down-shifted spectrum is observed. In a nondegenerate plasma, the Compton scattering spectrum will reflect a Maxwell-Boltzmann distribution, providing a measure of the electron temperature whereas for degenerate plasma, the Compton spectrum will reflect a Fermi distribution.

For $\alpha>1$, one has access to the collective regime and to the plasmon scattering features. For a classical collisionless plasma an expression for the plasmon dispersion has been given by Bohm and Gross [22]:

$$
\omega_{B G}^{2}=\omega_{p e}^{2}+\frac{3 k_{B} T_{e}}{m_{e}} k^{2}
$$

with $\omega_{p e}=\sqrt{\frac{4 \pi n_{e} e^{2}}{m_{e}}}$, the electronic plasma frequency. This relation has been obtained by expanding the dielectric function to second order in $k$ for a classical Maxwell-Boltzmann ideal plasma. In order to extend the range of applicability to higher wave numbers and higher densities (or lower temperatures), a modified Bohm-Gross dispersion relation including electron quantum diffraction effects has been proposed [17]:

$$
\omega_{B G m o d}^{2}=\omega_{p e}^{2}+\frac{3 k_{B} T_{e}}{m_{e}} k^{2}\left(1+0.088 n_{e} \Lambda_{e}^{3}\right)+\left(\frac{\hbar k^{2}}{2 m_{e}}\right)^{2} .
$$

These relations do not account for particle interactions. Their range of applicability is restricted to small $k$ where the interactions between particles are predominant. In our model, all the interactions between particles are taken into account but as noted in the previous section, degeneracy effects are missing. Using the same idea as for the modification of $\omega_{B G}$ and assuming that the differences between the RPA and RPA in classical limit are all due to degeneracy effects, we propose a modification of our pure classical results obtained with TCP-MD that combines the consideration of interactions between all particles and the effects of degeneracy. In practical terms, the difference between the two curves representing the maximum position of $S_{e e}(k, \omega)$ calculated in the RPA and the RPA in the classical limit models has been fitted by a polynomial function, $P(k)$, of the variable $k$. This polynomial function thus captures the effects of electron degeneracy, and the TCP-MD results have been modified as follows:

$$
\Delta E_{T C P-M D \text { mod }}^{2}=\Delta E_{T C P-M D}^{2}+P(k)^{2} .
$$

Comparisons of the results obtained by TCP-MD with RPA and RPA in the classical limit results and pure electron OCP simulations are plotted in Fig. 7 and in Fig. 8 for un-compressed and compressed cases, respectively.

Comparing the results obtained with pure classical models, it can be seen in Fig. 7 that the positions of the plasmon peaks in the TCP-MD and in the OCP are shifted towards lower frequencies relative to the RPA in the classical limit due to interactions between particles. For large $k$ ( $\operatorname{small} \alpha$ ), both RPA and simulations yield almost the same location for the maximum peak of the Compton spectrum. Here the non-collective regime has been reached and the Compton spectrum reflects the thermal electronic motion. 


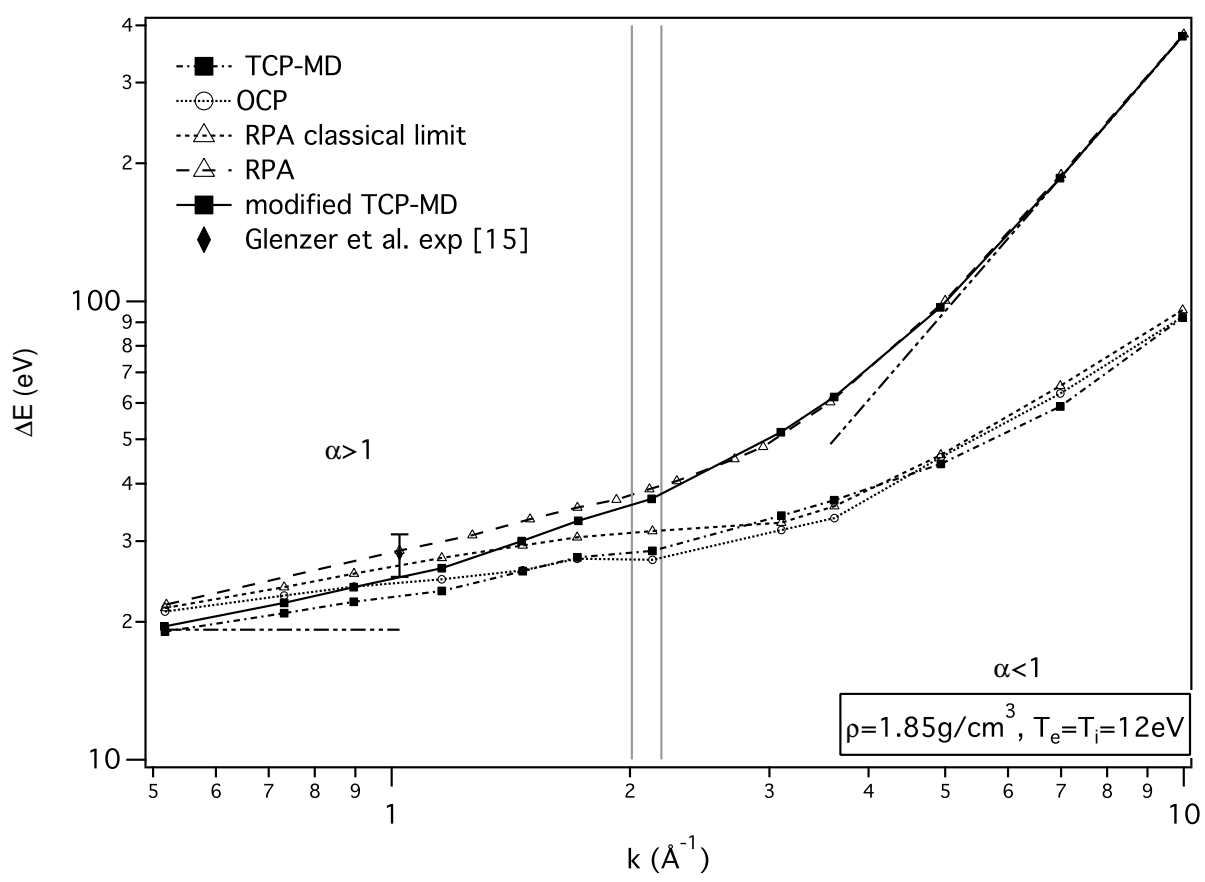

Figure 7: Maximum position $\Delta E$ of $S_{e e}(k, \omega)$ as a function of $k$ for the un-compressed case conditions. The vertical grey lines show from the left to the right, the positions of the inverse of $\lambda_{D}$ and $\lambda_{T F}$, respectively. The two dash-double dot lines at small and large $k$ show the two asymptotes.

When the degeneracy effects are accounting for, RPA (empty triangles and long dashes) and modified TCP-MD (black squares and full line) differ at low $k$ due to the strong influence of particle interactions, but they give similar results as soon as $1 / k \lambda_{T F}<1$.

Finally, for sake of completeness, we have plotted in Fig. 7 the maximum position of the plasmon peak (diamond) measured in the un-compressed beryllium experiment [15].

Similar results have been obtained for the compressed beryllium conditions. The results are plotted in Fig. 8. Owing to the fact that the parameter $\alpha$ does not span exactly the same range of values as previously, similar behavior is observed comparing the different results obtained with pure classical models. The maximum peak values obtained with the modified TCP-MD compare very well with the data points measured in the compressed beryllium experiment [16] plotted again for completeness.

To summarize, a two-component plasma classical molecular dynamics simulation code has been applied to calculate free electron dynamic structure factors for beryllium in warm dense matter conditions. The ionic structure properties compare well with results obtained with finite temperature density functional theory molecular dynamics and also compare well with the ionic mean charge and thus the electronic density. Concerning the electron dynamic properties, to neglect the degeneracy effects is a weakness of this model. When the scattering wavelength is such that the $\alpha$ parameter is much larger than unity, the electron degeneracy effects are negligible and our results are useful to investigate the role of interactions between particles and how these affect the position of the plasmon peak and the broadening of the structure factor. In order to extend the range of applicability of our results, a modification of the relation that gives the position of the maximum peak of the DSF has been proposed to keep the benefit of the classical MD while including the effects of electron degeneracy, which is important as estimates for the peak position are useful for plasma diagnostics.

\section{References}

[1] S.H. Glenzer, R. Redmer, Review of Modern Physics 81 (2009) 1625 (39).

[2] A. Calisti, S. Ferri, B. Talin, HEDP 5 (2009) 307. 


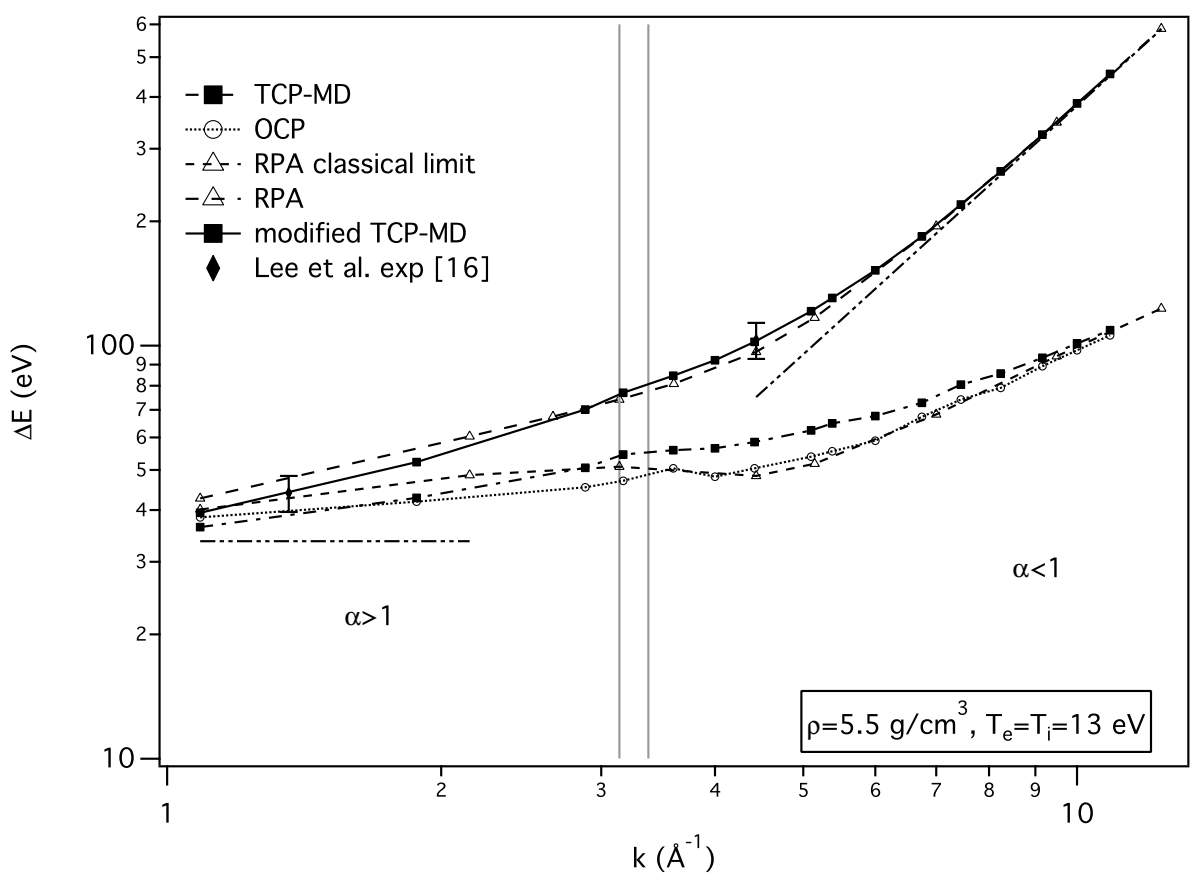

Figure 8: Same as Fig. 7 for the compressed case conditions.

[3] A. Calisti, B. Talin, Contrib. Plasma Phys. 516 (2011) 524-528.

[4] J.P. Hansen, I.R. McDonald, Phys. Rev. A 23 (1981) 2041.

[5] A.V. Filinov et al., Phys. Rev. E 70 (2004) 046411

[6] A. Calisti et al., HEDP 7 (2011) 197-202.

[7] W.D. Kraeft, D. Kremp, W. Ebeling, and R. Röpke, Quantum Statistics of Charged Particle Systems, (Akademie-Verlag, Berlin, 1986).

[8] S. Ichimaru, Statistical Plasma Physics: I. Basic Principles, (Addison-Wesley, Reading, 1992); Statistical Plasma Physics II: Condensed Plasmas, (Addison-Wesley, Reading, 1994).

[9] H. Minoo, M.M. Gombert, C. Deutsch, Phys. Rev. A 23 (1981) 924.

[10] F.J. Rogers, Phys. Rev. A 23 (1981) 1008-1014.

[11] C.S. Jones, M.S. Murillo, HEDP 3 (2007) 379-394.

[12] I.I. Sobelman, L.A. Vainstein, E.A. Yukov, Excitation of Atoms and Broadening of Spectral Lines, Springer Series in Chemical Physics 7, ed. V.I. Goldanskii et al., Springer-Verlag Berlin Heidelberg New-York 1981

[13] K. U. Plagemann et al., New Journal of Physics 14 (2012) 055020.

[14] E.E. Salpeter, Phys. Rev. 120 (1960) 1528.

[15] S.H. Glenzer et al., Phys. Rev. Lett. 98 (2007) 065002.

[16] H.J. Lee et al., Phys. Rev. Lett. 102 (2009) 115001.

[17] R. Thiele et al., Phys. Rev. E 78 (2008) 026411.

[18] K. Wünsch, J. Vorberger, D.O. Gericke, Phys. Rev. E 79 (2009) 010201(R)

[19] V. Schwarz et al., HEDP 6 (2010) 305.

[20] J. Ye, B. Zhao, J. Zheng, Phys. Plasmas 18 (2011) 032701.

[21] N.R. Arista and W. Brandt, Phys. Rev. A 29 (1984) 1471.

[22] D. Bohm, E.P. Gross, Phys. Rev. 75 (1949) 1851. 